\title{
Could S6K1 immunopositivity be used to distinguish early and advanced stages of endometrioid endometrial adenocarcinoma?
}

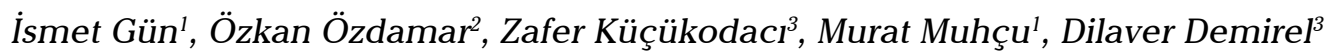 \\ ${ }^{1}$ Department of Obstetrics and Gynecology, Gülhane Military Medical Academy, Haydarpaşa Training and Research \\ Hospital, Istanbul, Turkey \\ ${ }^{2}$ Department of Obstetrics and Gynecology, İstanbul Medeniyet University School of Medicine, İstanbul, Turkey \\ ${ }^{3}$ Department of Pathology, Gülhane Military Medical Academy, Haydarpaşa Training and Research Hospital, İstanbul, Turkey
}

\section{Abstract}

Objective: To assess whether the immunopositivity of S6K1, a crucial effector of the mTOR signaling pathway, varies between early-stage low-grade and advanced-stage high-grade endometrial endometrioid adenocarcinoma (EEA) as well as to discuss its prognostic significance.

Material and Methods: A total of 22 normal endometrial tissue samples (Control group) and 41 EEA specimens (Study group) were enrolled in the study, and all the samples underwent immunohistochemical staining for S6 kinase alpha (S6K1). The study group was further evaluated in two subgroups; stage 1A, grade 1 (Group 1) and stage $\geq 1 \mathrm{~A}$, grade 2 or 3 (Group 2). Group 2 patients were considered as a poor prognosis for EEA. The samples were examined by two independent pathologists. Statistical analyses were performed using the Student's t-test for continuous variables, the Chi-square test for categorical variables, and one-way analysis of variance for the comparison of multiple variables.

Results: The immunopositivity rate for all the included EEA patients was $56.1 \%$, whereas none of the 22 normal endometrial tissue samples revealed immunoreactivity for S6K1. The immunopositivity rates were significantly different between Groups 1 and 2 [38.1\% (8/21) and 75.0\% $(15 / 20)$, respectively, $\mathrm{p}=0.039$ ]. When $\mathrm{S} 6 \mathrm{~K} 1$ positivity was used as a criterion of poor prognosis in EEA, the sensitivity, specificity, positive predictive value, and negative predictive value were calculated to be $62 \%, 75 \%, 72 \%$, and $65 \%$, respectively (OR: 4.9 and $95 \%$ CI: $1.3-18.7$ ).

Conclusion: S6K1 was positive in the majority of EEAs and malignancies at an advanced stage. Higher grade disease had a significantly higher rate of S6K1 positivity. S6K1 immunopositivity appears to be a promising method to predict poor prognosis in EEA.

(J Turk Ger Gynecol Assoc 2016; 17: 163-7)

Keywords: Endometrioid endometrial adenocarcinoma, P70 ribosomal protein S6 kinase alpha, PI3 K/AKT/mTOR pathway, prognostic indicator Received: 11 April, 2016 Accepted: 28 June, 2016

\section{Introduction}

Endometrial cancer (EC) is the most common gynecological malignancy in developed countries, with the most frequent histological type being endometrial endometrioid adenocarcinoma (EEA), which accounts for $70 \%-80 \%$ of all cases (1). EEAs are different from non-EEAs in molecular and clinical pathology and are associated with less aggressive behavior and better prognosis as well as earlier diagnosis (2). Thus, a surgical approach is sufficient in early-stage EEA; however, adjuvant treatment is recommended for women with a moderate to high risk of recurrence $(3,4)$. Patients with advanced or recurrent disease have a poor median survival; therefore, cytotoxic or radiation therapy should accompany surgery in those with invasion of more than $50 \%$ of myometrium or with advanced disease, including early stage at grade 2 or 3 . It appears clinically important to identify the markers that determine the need for adjuvant therapy to surgery and that predict a poor prognosis in patients with EEA. Moreover, novel therapeutic approaches, such as molecular targeted therapy, which have a lower side-effect profile and systemic toxicity, are necessitated, particularly in patients with advanced or recurrent disease $(5,6)$. The mammalian target of the rapamycin (mTOR) signaling pathway is directly involved in many cell signaling pathways and mainly regulates three important downstream substrates: eukaryotic initiation factor 4E (eIF4E), eukaryotic translation initiation factor 4E-binding protein 1 (4EBP1), and P70 ribosomal protein S6 kinase alpha (S6K1) (5-7). Activation of the mTOR signaling pathway and aberrations of the mTOR, including PI3K amplification/mutation and S6K1 overexpression, have been reported in a set of malignancies and in some types of gynecologic cancers $(8,9)$. In the present study, our aim was to investigate the expression patterns of $\mathrm{S} 6 \mathrm{~K} 1$, a crucial effector of mTOR signaling, in tis- 
sues of non-pathologic endometrium, early-stage (IA, Grade 1) and advanced-stage (IA, grade 2-3, and IB, II, III, IV) EEA, using indirect immunohistochemistry.

\section{Material and Methods}

\section{Clinical samples}

This is a cross-sectional study conducted between January 2003 and December 2011. Ethical approval from the Institutional Review Board was granted before the initiation. Patients who underwent surgery for EEA and in whom diagnosis was made upon pathological examination, were extracted retrospectively from the clinic's patient database. The control group consisted of patients undergoing surgery for non-endometrial benign gynecological diseases. Patients who had received treatment that could potentially affect S6K1 immunostaining, such as chemotherapy, radiotherapy, and hormone replacement therapy (HRT) or oral contraceptive pills, and those with concurrent malignancies, cardiac hypertrophy, and type 2 diabetes and obesity, were excluded from the study. All the specimens were re-evaluated by a single pathologist, and all the pathological diagnoses were confirmed by another pathologist before the study. During these examinations, cases without sufficient tissue samples were excluded. Patients diagnosed as EEA by pathological examination were divided into two subgroups: Group 1; grade 1, stage 1A EEA patients, and, Group 2; grade 2 or 3, stage $\geq 1 \mathrm{~A}$ EEA patients. Stages for endometrial cancer were determined according to the clinical criteria established by the International Federation of Gynecology and Obstetrics (FIGO) 2014 (10). Clinical and pathology data from the included patients were recorded.

\section{Details of the antibody used and analysis of the immunohistochemistry}

4 micron thick cross-sections were taken from suitable paraffin blocks. These cross-sections, in conjunction with positive controls, were incubated for 17 hours at $55^{\circ} \mathrm{C}$, and, after standard deparaffinization and rehydration processes, were applied. Afterwards, the immunohistochemical staining process was manually performed in accordance with the suggested procedure with a 1/100 dilution of polyclonal Rabbit P70S6K1 (Anti-S6K1 antibody (ab47504), Abcam; Cambridge, MA, USA) primer antibody. HeLa cells were used as positive control materials.

\section{Evaluation of the staining}

The immunohistochemical results were evaluated under a microscope independently by two pathologists who did not have any knowledge of the clinical outcome and who scored the results semi-quantitatively. Any discrepancies between the staining intensity score results between the two pathologists were noted and referred to as the interobserver difference. We used a scoring method referred to as the expression index (EI), which was based on two characteristics: overall stain intensity and the percentage of neoplastic tissue that was stained. The staining intensity was scored as 0 (no staining), 1+ (weakly positive), $2+$ (moderately positive), 3+ (strongly positive). The most frequently observed scores were recorded for areas painted in varying intensities. An EI was calculated by multiplying the staining intensity score and the percentage of positively stained cells (ranging from 0 to 300 ).

Then, the resulting staining intensity scores and the percentage of positive staining were averaged between the two pathologists and were recorded. In the present study, the interobserver differences were less than $5 \%$.

\section{Statistical analysis}

Statistical analysis was performed using the Statistical Package for the Social Sciences for Windows 15.0 software (SPSS Inc.; Chicago, IL, USA). Descriptive statistics were given as the mean, standard deviation, frequency, and percentage. Statistical analysis was performed using the Student's t-test for comparison of two continuous variables, one-way ANOVA for comparison of multiple variables, and the $\mathrm{x}^{2}$ test for categorical variables. When there was a need for a non-parametric test, the MannWhitney U and Kruskal-Wallis tests were performed. Statistical significance was defined as $\mathrm{p}<0.05$. A nominal two-sided $\mathrm{p}$ value was considered for all comparisons.

\section{Results}

Immunohistochemical staining for S6K1 expression was performed on paraffin-embedded biopsy specimens from a total of 63 patients, including 22, 21, and 20 cases in the control group and Groups 1 and 2, respectively. Of all the EEA cases, 56.1\% were immunostaining positive, whereas none of the 22 normal gynecologic endometrial tissue samples revealed immunoreactivity for S6K1. Figures 1 and 2 show the activation status of immunocytochemical staining for S6K1, whereas Figure 3 shows the EI status of $\mathrm{S} 6 \mathrm{~K} 1$ protein in all groups. Comparisons of the patient age and $\mathrm{EI}$ and $\mathrm{S} 6 \mathrm{~K} 1$ results between the groups are presented in Table 1. Accordingly, EI was higher in early-stage patients than in advanced-stage patients, but not very significant $(p=0.107)$. The percentage of S6K1 immunopositive tissues was significantly higher in advanced-stage EEAs than in early-stage EEAs ( $75 \%$ vs. $38.1 \%$, respectively; $p=0.039$ ), indicating that as the stage of the disease advanced, the chance of having a positive immunostaining increased. When $\mathrm{S} 6 \mathrm{~K} 1$ positivity was used as a criterion of advanced stage in EEA, the sensitivity, specificity, positive predictive value, and negative predictive value were calculated to be $62 \%, 75 \%, 72 \%$, and $65 \%$, respectively (OR: 4.9 and 95\% CI: 1.3-18.7).

Table 1. Comparisons between the groups in terms of age and EI and S6K1 immunostaining

\begin{tabular}{|c|c|c|c|}
\hline Characteristics & Group $1(n=21)$ & Group $2(n=20)$ & $\mathbf{p}$ \\
\hline Age, years & $62 \pm 10.8$ & $66.3 \pm 9.3$ & 0.189* \\
\hline EI & $17.6 \pm 49.2$ & $14.1 \pm 24.8$ & $0.107^{*}$ \\
\hline S6K1 immunostaining (-) & $13(31 \%)$ & $5(12 \%)$ & \\
\hline S6K1 immunostaining $(+)$ & $8(20 \%)$ & $15(37 \%)$ & $0.039 * *$ \\
\hline \multicolumn{4}{|c|}{$\begin{array}{l}\text { Data are presented as the mean } \pm \text { standard deviation and number (percent) } \\
\text { The p value was determined by using *Mann-Whitney U test and } \\
* * \text { Chi-square test. }\end{array}$} \\
\hline
\end{tabular}




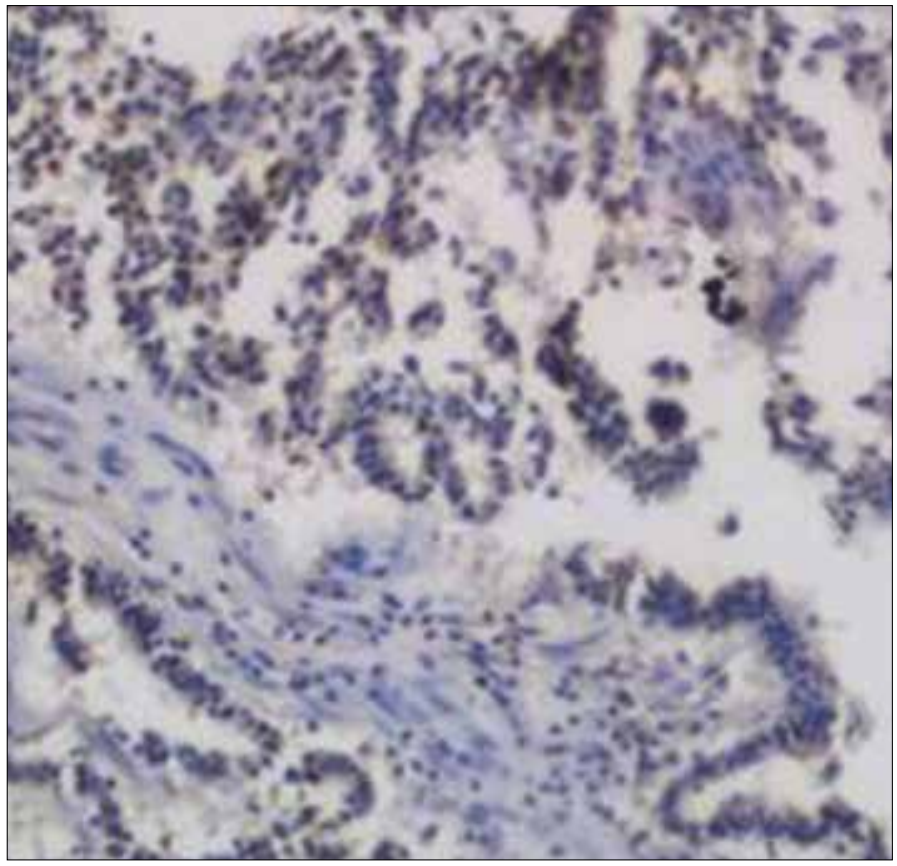

Figure 1. Activation status of immunocytochemical staining for P70S6K1 in endometrial endometrioid adenocarcinoma. Weak staining with $\times 200$ magnification.

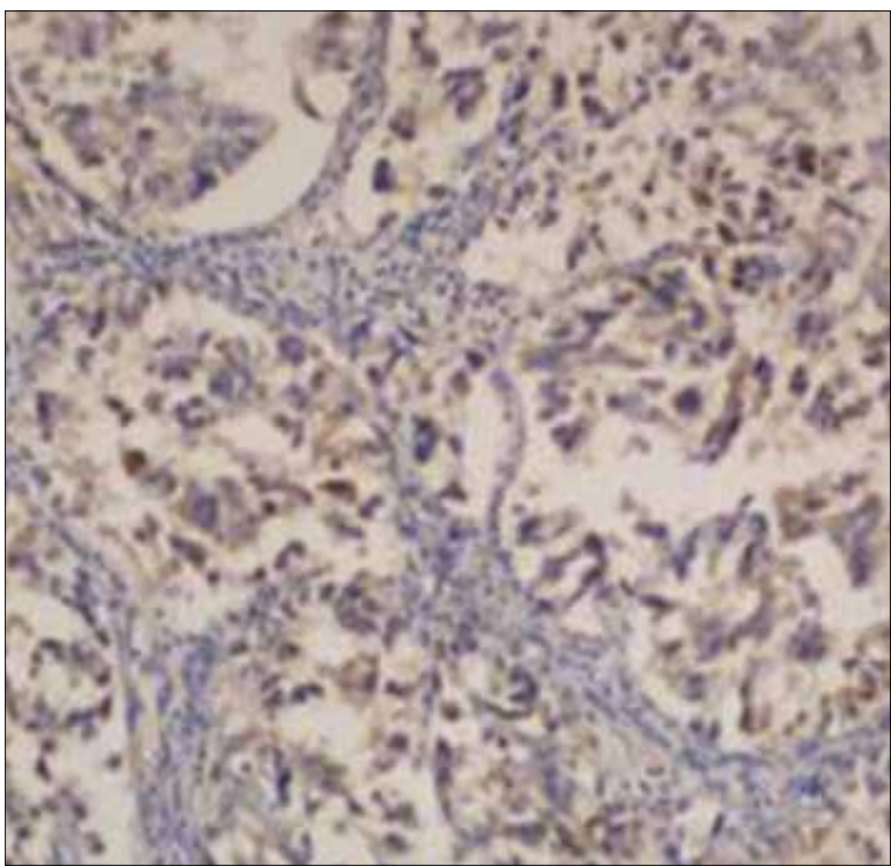

Figure 2. Activation status of immunocytochemical staining for P70S6K1 in endometrial endometrioid adenocarcinoma. Moderate staining with $\times 100$ magnification.

\section{Discussion}

In this immunohistochemistry study, we examined whether S6K1, a downstream target of mTOR, was activated in EEA and whether its expression correlated with disease stage. There was no S6K1 immunostaining positivity in any specimen of the 22

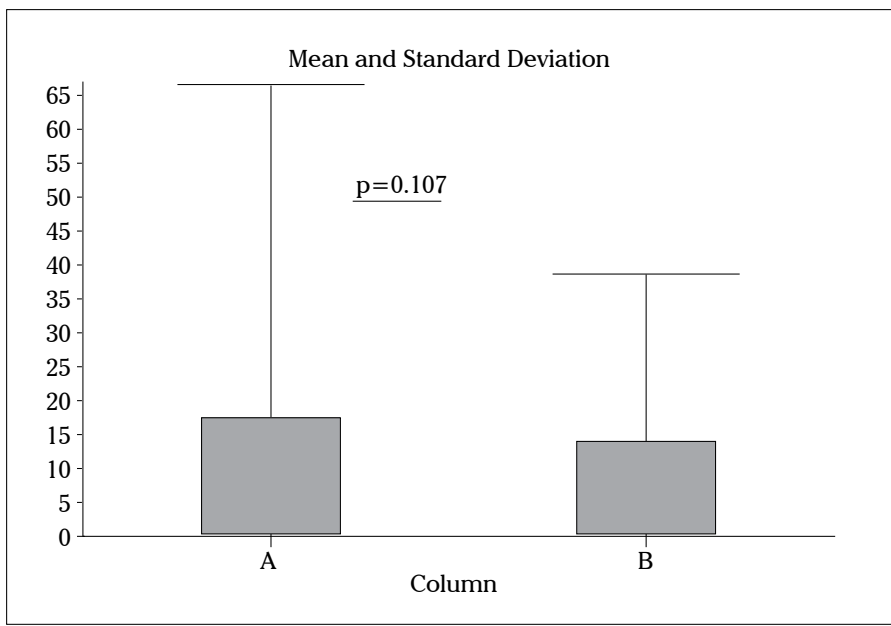

Figure 3. Expression index status of S6K1 protein in Group 1 and Group 2

normal tissue-containing group. However, S6K1 immunopositivity rates in Groups 1 and 2 were $38.1 \%$ and 75\%, respectively $(\mathrm{p}=0.039)$, suggesting that the percentage of S6K1 immunopositive tissues was significantly higher in advanced-stage EEAs than in early-stage EEAs. Tumors positive for S6K1 expression were significantly more likely to be at an advanced stage than those negative for S6K1 staining, indicating that as the stage of the disease advanced, the chance of having a positive immunostaining result increased. The sensitivity and specificity rates of S6K1 immunostaining positivity in determining poor prognosis were $62 \%$ and $75 \%$, respectively.

Inter- and intra-cellular signaling is essential for the maintenance of cellular life. Protein kinases regulate many cell signaling pathways involved in protein phosphorylation/activation (11). Serine/threonine kinases constitute an important group in cytoplasmic protein kinases. Phosphatase and tensin homologue deleted on chromosome ten (PTEN)/phosphatidylinositol 3-kinase (PI3K)/AKT/mTOR is considered to be a crucial regulatory pathway of protein translation, and mTOR is a $289 \mathrm{kDa}$ serine/threonine protein kinase located in the center of this pathway (12). mTOR exists in two distinct complexes: mTORC1 and mTORC2. mTORC1 is sensitive to rapamycin and is regulated by multiple signals, such as growth factor, nutrients, energy status, oxygen, and cellular stress. mTORC1 phosphorylates and activates $\mathrm{S} 6 \mathrm{~K} 1$ and 4EBP1, which in turn, enhances protein synthesis, proliferation, cell survival, ribosome biogenesis, angiogenesis, migration, invasion, and metastasis. (8, 12, 13).

The deregulation of multiple elements of the mTOR pathway, such as amplification or mutation of PI3K, loss of PTEN function, and overexpression of AKT, S6K1, 4EBP1, and eIF4E, has been reported in numerous types of human diseases, including cancer, cardiac hypertrophy, type 2 diabetes, and obesity. $(8,12,14)$. Aberrations of the mTOR signaling pathway are common in several types of human cancers, such as breast, ovarian, prostate, bladder, thyroid, colon, and head and neck cancers (8, 15-18). Activation of the PI3K/AKT/mTOR pathway and increased mTOR signaling have been reported to be associated with chemoresistance, aggressive disease, and poor prognosis in many cancer types, irrespective of the tumor type (19-23). In vivo studies support the 
hypothesis that a loss of PTEN and subsequent AKT activation result in the activation of Estrogen Receptor- $\alpha(E R \alpha)$-dependent pathways that play a pivotal role in the neoplastic process (24). In a recent study, nuclear phosphorylated (p) Ser(167)-ER $\alpha$ was reported to significantly positively correlated with p-MAPK and pS6K1 and with a significantly shorter relapse-free survival in EEA (25). Another study reported that extracellular signal-regulated kinase (ERK1/2) and p-AKT can be useful in the differential diagnosis of benign vs. malignant endometrial lesions as well as early- vs. advanced-stage EEA (26). Similarly, our results suggest that $\mathrm{S} 6 \mathrm{~K} 1$ immunopositivity could be used as a predictive test in EEAs and is a promising prognostic indicator of advanced stage and higher grade disease. On the other hand, mTOR inhibition has been associated with the diminished development and progression of hyperplastic lesions (27) in endometrial cancer cell lines. To date, six phase II trials assessing the use of rapalogs in recurrent endometrial carcinoma have been published (28). Surprisingly, two studies conducted in patients with endometrial cancer revealed no statistically significant correlation between activity of the PI3K/AKT/mTOR pathway and clinicopathological characteristics, including stage, grade, and lymph node involvement. $(29,30)$. In a very recent study, S6K1 expression has been reported to be a promising biomarker of sensitivity (31). Based on the results of our study, we demonstrated that only malignant tissue cell lines are associated with $\mathrm{S} 6 \mathrm{~K} 1$ immunostaining, whereas benign pathologies are not. Moreover, S6K1 expression is associated with advanced stage and a poor prognosis of disease. Given the uncertainty with respect to the adjuvant therapy options and fertility-sparing surgery in women with stage IA, grade 2 disease, S6K1 may cease the scientific debate as an indicator of poor prognosis. S6K1 immunostaining tests in women with EEA might surrogate advanced stage and poor prognosis and hence may be used to determine the appropriate therapeutic approach in this patient population.

In our study, EI, which is a scoring method based on overall stain intensity and the percentage of neoplastic tissue that is stained, was higher in early-stage patients than in advanced-stage patients, but not significantly so $(p=0.107)$. Even if pathological specimens were evaluated independently by two pathologists in a blinded fashion and their results were averaged to minimize interobserver variability, the semi-quantitative scoring method might have influenced the results. Studies investigating the relationship between staining patterns and the stage or grade of the disease with larger patient numbers and using quantitative interpretation methods may indicate significant differences.

There is a very limited number of studies in the available literature investigating the mTOR pathway, S6K1 signaling, and endometrial carcinomas, which thus constitutes a substantial strength of this study. However, our study is limited by a number of matters. First, the number of the patients in our study was relatively low. The second is the lack of the treatment outcomes and survival period of the included patients. In this study, we excluded patients with certain clinical conditions, such as type 2 diabetes and obesity, which are well-known risk factors for EEA. This situation might be perceived to have biased the results of the study. However, it has been known that the mTOR pathway is mediated by a wide variety of cellular signal communications, which include hormones, such as insulin and growth factors; nutrients, such as amino acids and glucose; and cellular stress conditions. mTOR integrates signals from a variety of "energy balancing" hormones, such as leptin, insulin, and ghrelin, although its action varies in response to these distinct hormonal stimuli as well as across different neuronal populations, and it has roles in the regulation of body weight, energy expenditure, and glucose/lipid homeostasis (32). Moreover, the mTOR pathway has been associated with obesity in numerous studies, in which the risk of obesity was correlated with the overactivation of the mTOR-Raptor-S6K1 signaling pathway and a decrease in AKT levels. On the other hand, insulin resistance is a major aspect of type 2 diabetes, which results from impaired insulin signaling in target cells, and it was reported that the mTORC2mediated phosphorylation of PKB-Ser473 was unperturbed in type 2 diabetes (33). From this aspect, we intended to avoid the confounding effects of clinical situations that may potentially interact with the mTOR pathway, such as obesity and type 2 diabetes, and to evaluate the association between endometrium carcinoma and S6K1 expression.

In conclusion, although S6K1 immunostaining was weak-moderate in both early and advanced stages, advanced stage and higher grade disease is significantly more likely to reveal S6K1 immunopositivity than early-stage, low-grade disease. S6K1 immunopositivity appears to be a promising method to predict poor prognosis in EEA. There is a need for more comprehensive studies on this subject.

Ethics Committee Approval: Ethics committee approval was received for this study from the ethics committee of Gülhane Military Medical Academy, Haydarpaşa Training and Research Hospital (11.11.2010, Project Number: 101).

Informed Consent: Written informed consent was obtained from patients who participated in this study.

Peer-review: Externally peer-reviewed.

Author Contributions: Concept - I.G., Ö.Ö.; Design - I.G., Ö.Ö.; Supervision - I.G., M.M.; Resources - Ö.Ö., Z.K.; Materials - Ö.Ö., Z.K.; Data Collection and/or Processing - I.G., Ö.Ö.; Analysis and/or Interpretation - I.G., D.D.; Literature Search - Ö.Ö., Z.K.; Writing Manuscript - Ö.Ö., I.G.; Critical Review - M.M., D.D.

Conflict of Interest: No conflict of interest was declared by the authors.

Financial Disclosure: The authors declared that this study has received no financial support.

\section{References}

1. Jemal A, Siegel R, Ward E, Hao Y, Xu J, Thun MJ. Cancer statistics. CA Cancer J Clin 2009; 59: 225-49. [Crossref]

2. Amant F, Moerman P, Neven P, Timmerman D, Van LE, Vergote I. Endometrial cancer. Lancet 2005; 366: 491-505. [Crossref]

3. Cengiz M, Singh AK, Grigsby PW. Postoperative vaginal brachytherapy alone is the treatment of choice for grade 1-2, stage IC endometrial cancer. Int J Gynecol Cancer 2005; 15: 926-31. [Crossref]

4. Horowitz NS, Peters WA $3^{\text {rd }}$, Smith MR, Drescher CW, Atwood M, Mate TP. Adjuvant high dose rate vaginal brachytherapy as treatment of stage I and II endometrial cancer. Obstet Gynecol 2002; 99: 235-40. [Crossref] 
5. Thanapprapasr D, Thanapprapasr K. Molecular therapy as a future strategy in endometrial cancer. Asian Pac J Cancer Prev 2013; 14: 3419-23. [Crossref]

6. Umene K, Banno K, Kisu I, Yanokura M, Nogami Y, Tsuji K, et al. New candidate therapeutic agents for endometrial cancer: potential for clinical practice (review). Oncol Rep 2013; 29: 855-6.

7. Dancey J. mTOR signaling and drug development in cancer. Nat Rev Clin Oncol 2010; 7: 209-19. [Crossref]

8. Pópulo H, Lopes JM, Soares P. The mTOR Signalling Pathway in Human Cancer. Int J Mol Sci 2012; 13: 1886-918. [Crossref]

9. Xu G, Zhang W, Bertram P, Zheng XF, McLeod H. Pharmacogenomic profiling of the PI3K/PTENAKT-mTOR pathway in common human tumors. Int J Oncol 2004; 24: 893-900.

10. FIGO Committee on Gynecologic Oncology. FIGO staging for carcinoma of the vulva, cervix, and corpus uteri. Int $\mathrm{J}$ Gynaecol Obstet 2014; 125: 97-8. [Crossref]

11. Manning G, Whyte DB, Martinez R, Hunter T, Sudarsanam S. The protein kinase complement of the human genome. Science 2002; 298: 1912-34. [Crossref]

12. Zhou H, Huang $\mathrm{S}$. The complexes of mammalian target of rapamycin. Curr Protein Pept Sci 2010; 11: 409-24. [Crossref]

13. Zoncu R, Efeyan A, Sabatini DM. mTOR: from growth signal integration to cancer, diabetes and ageing. Nat Rev Mol Cell Biol 2011; 12: 21-35. [Crossref]

14. Djordjevic B, Hennessy BT, Li J, Barkoh BA, Luthra R, Mills GB, et al. Clinical assessment of PTEN loss in endometrial carcinoma: immunohistochemistry outperforms gene sequencing. Mod Pathol 2012; 25: 699-708. [Crossref]

15. Xing M. Genetic alterations in the phosphatidylinositol-3 kinase/Akt pathway in thyroid cancer. Thyroid 2010; 20: 697-706. [Crossref]

16. Nawroth R, Stellwagen F, Schulz WA, Stoehr R, Hartmann A, Krause $\mathrm{BJ}$, et al. S6K1 and 4E-BP1 are independent regulated and control cellular growth in bladder cancer. PLoS One 2011; 6: e27509. [Crossref]

17. Gao W, Li JZ, Chan JY, Ho WK, Wong TS. mTOR Pathway and mTOR Inhibitors in Head and Neck Cancer. ISRN Otolaryngol 2012; 2012: 953089. [Crossref]

18. Karlsson E, Pérez-Tenorio G, Amin R, Bostner J, Skoog L, Fornander $\mathrm{T}$, et al. The mTOR effectors 4EBP1 and S6K2 are frequently coexpressed, and associated with a poor prognosis and endocrine resistance in breast cancer: a retrospective study including patients from the randomised Stockholm tamoxifen trials. Breast Cancer Res 2013; 15: R96. [Crossref]

19. Kremer CL, Klein RR, Mendelson J, Browne W, Samadzedeh LK, Vanpatten $\mathrm{K}$, et al. Expression of mTOR signaling pathway markers in prostate cancer progression. Prostate 2006; 66: 1203-12. [Crossref]

20. Faried LS, Faried A, Kanuma T, Aoki H, Sano T, Nakazato T, et al. Expression of an activated mammalian target of rapamycin in adenocarcinoma of the cervix: A potential biomarker and molecular target therapy. Mol Carcinog 2008; 47: 446-57. [Crossref]

21. Faried LS, Faried A, Kanuma T, Sano T, Nakazato T, Tamura T, et al. Predictive and prognostic role of activated mammalian target of rapamycin in cervical cancer treated with cisplatin-based neoadjuvant chemotherapy. Oncol Rep 2006; 16: 57-63. [Crossref]

22. Salvesen HB, Carter SL, Mannelqvist M, Dutt A, Getz G, Stefansson IM, et al. Integrated genomic profiling of endometrial carcinoma associates aggressive tumors with indicators of PI3 kinase activation. Proc Natl Acad Sci U S A 2009; 106: 4834-9. [Crossref]

23. Darb-Esfahani S, Faggad A, Noske A, Weichert W, Buckendahl AC, Müller B, et al. Phospho-mTOR and phospho-4EBP1 in endometrial adenocarcinoma: association with stage and grade in vivo and link with response to rapamycin treatment in vitro. $\mathrm{J}$ Cancer Res Clin Oncol 2009; 135: 933-41. [Crossref]

24. Vilgelm A, Lian Z, Wang H, Beauparlant SL, Klein-Szanto A, Ellenson $\mathrm{LH}$, et al. Akt-mediated phosphorylation and activation of estrogen receptor alpha is required for endometrial neoplastic transformation in Pten+/- mice. Cancer Res 2006; 66: 3375-80. [Crossref]

25. Kato E, Orisaka M, Kurokawa T, Chino Y, Fujita Y, Shinagawa A, et al. Relation between outcomes and expression of estrogen receptor- $\alpha$ phosphorylated at Ser(167) in endometrioid endometrial cancer. Cancer Sci 2014; 105: 1307-12. [Crossref]

26. Gungorduk K, Ertas IE, Sahbaz A, Ozvural S, Sarica Y, Ozdemir A, et al. Immunolocalization of ERK1/2 and p-AKT in normal endometrium, endometrial hyperplasia, and early and advanced stage endometrioid endometrial adenocancer and their prognostic significance in malignant group. Eur J Obstet Gynecol Reprod Biol 2014; 179: 147-52. [Crossref]

27. Milam MR, Celestino J, Wu W, Broaddus RR, Schmeler KM, Slomovitz BM, et al. Reduced progression of endometrial hyperplasia with oral mTOR inhibition in the PTEN heterozygote murine model. Am J Obstet Gynecol 2007; 196: 247.e1-5. [Crossref]

28. Lheureux S, Wilson M, Mackay H. Recent and current phase II clinical trials in endometrial cancer: review of the state of art. Expert Opin Investigating Drugs 2014; 23: 773-92. [Crossref]

29. No JH, Jeon YT, Park IA, Kang D, Kim JW, Park NH, et al. Expression of mTOR protein and its clinical significance in endometrial cancer. Med Sci Monit 2009; 15: BR301-5.

30. Mori N, Kyo S, Sakaguchi J, Mizumoto Y, Ohno S, Maida Y, et al. Concomitant activation of AKT with extracellular-regulated kinase 1/2 occurs independently of PTEN or PIK3CA mutations in endometrial cancer and may be associated with favorable prognosiss. Cancer Sci 2007; 98: 1881-8. [Crossref]

31. Santacana M, Coronado P, Matias-Guiu X, Romero I, Casado A, GilMoreno A, et al. Biological Effects of Temsirolimus on the mTOR Pathway in Endometrial Carcinoma: A Pharmacodynamic Phase II Study. Int J Gynecol Cancer 2016. [Crossref]

32. Hu F, Xu Y, Liu F. Hypothalamic Roles of mTOR Complex I: Integration of nutrient and hormone signals to regulate energy homeostasis. Am J Physiol Endocrinol Metab 2016. [Crossref]

33. Rajan MR, Nyman E, Kjolhede P, Cedersund G, Stralfors P. SystemsWide Experimental and Modeling Analysis of Insulin Signaling through FOXO1 in Human Adipocytes, Normally and in Type 2 Diabetes. J Biol Chem 2016. [Crossref] 